\title{
Age-related changes in visual encoding strategy preferences during a spatial memory task
}

\author{
Vladislava Segen $^{1,2,5} \cdot$ Marios N. Avraamides ${ }^{3,4} \cdot$ Timothy J. Slattery $^{2} \cdot$ Jan M. Wiener ${ }^{1,2}$
}

Received: 3 October 2020 / Accepted: 16 February 2021 / Published online: 23 March 2021

(c) The Author(s) 2021

\begin{abstract}
Ageing is associated with declines in spatial memory, however, the source of these deficits remains unclear. Here we used eye-tracking to investigate age-related differences in spatial encoding strategies and the cognitive processes underlying the age-related deficits in spatial memory tasks. To do so we asked young and older participants to encode the locations of objects in a virtual room shown as a picture on a computer screen. The availability and utility of room-based landmarks were manipulated by removing landmarks, presenting identical landmarks rendering them uninformative, or by presenting unique landmarks that could be used to encode object locations. In the test phase, participants viewed a second picture of the same room taken from the same $\left(0^{\circ}\right)$ or a different perspective $\left(30^{\circ}\right)$ and judged whether the objects occupied the same or different locations in the room. We found that the introduction of a perspective shift and swapping of objects between encoding and testing impaired performance in both age groups. Furthermore, our results revealed that although older adults performed the task as well as younger participants, they relied on different visual encoding strategies to solve the task. Specifically, gaze analysis revealed that older adults showed a greater preference towards a more categorical encoding strategy in which they formed relationships between objects and landmarks.
\end{abstract}

\section{Introduction}

Successful navigation and orientation depend on our ability to recognise familiar places across different perspectives (Waller \& Nadel, 2013). In the lab, this ability is typically assessed with tasks in which participants first encode an array of objects or environmental features from one perspective and are then asked to indicate whether the array has changed when presented from a different perspective. Studies using such paradigms have reported age-related declines in performance (Hartley et al., 2007; Hilton et al.,

Vladislava Segen

vladislava.segen@gmail.com

1 Ageing and Dementia Research Centre, Bournemouth University, Bournemouth, UK

2 Department of Psychology, Bournemouth University, Bournemouth, UK

3 Department of Psychology, University of Cyprus, Nicosia, Cyprus

4 CYENS Centre, Nicosia, Cyprus

5 Aging and Cognition Research Group, German Center for Neurodegenerative Diseases (DZNE), Magdeburg, Germany
2020; Montefinese et al., 2015; Muffato et al., 2019; Segen et al., 2020). Building on these studies, and to gain a more detailed understanding of the factors that contribute to the performance decline, we use eye-tracking to investigate potential age-related differences in visual encoding strategies. Specifically, we are interested in whether young and older adults rely on the same or different environmental cues during place recognition.

Recently, Muffato et al. (2019) and Hilton et al. (2020) investigated the effects of cognitive ageing on place recognition abilities using scenes defined by objects that were placed in an open field. After encoding a scene with four objects, participants were presented with another scene from a different perspective and had to decide whether or not it was identical to the one encoded. Results revealed the presence of object-location binding errors, particularly in older adults. That is, compared to younger participants, older adults found it harder to detect that two objects had swapped locations than when one of the objects was replaced with a new object.

In our previous work (Segen et al., 2020), we investigated age-related differences in the ability to recognise spatial configurations across different perspectives. The task required participants to encode the locations of an array 
of identical objects presented as an image on a computer screen. The objects were arranged in clusters of one, two and three objects, in a virtual room containing additional environmental cues such as windows and a door. Then, participants viewed a second image of the same room taken from the same $\left(0^{\circ}\right)$ or a different perspective $\left(45^{\circ}\right.$ or $\left.135^{\circ}\right)$ and judged whether or not the objects were in the same locations. The positions of the objects were either changed by swapping two object clusters or by rotating one of the clusters. While with the former manipulation the task could be solved using a coarse categorical representation of the spatial relationships between object clusters (e.g. the cluster with two objects is to the left of the single object), the latter manipulation required a fine-grained spatial representation of the exact positions of the objects as the overall relationships between the clusters was maintained.

Consistent with previous research, we found that older adults had greater difficulty with the task than younger adults (Hartley et al., 2007; Hilton et al., 2020; Montefinese et al., 2015; Muffato et al., 2019). Diffusion modelling showed that older adults not only had greater difficulty in extracting useful information from the stimuli but that they also adopted a more conservative response strategy, i.e. they accumulated more information before reaching a decision.

Furthermore, the analysis of gaze data in Segen et al. (2020) revealed that older adults attended to a larger proportion of the scenes compared to younger adults. We proposed two potential explanations for this. First, differences in gaze behaviour may reflect differences in encoding strategies with older adults encoding object locations relative to the landmarks available in the room (windows, door, etc.), whilst young adults focus on the local arrangement of objects and on encoding the spatial relationships among them. The differences in encoding strategies may reflect a shift towards categorical spatial representations in older adults, driven by age-related hippocampal neurodegeneration (Antonova et al., 2009; Meulenbroek et al., 2004; Moffat et al., 2007).

Second, older adults may have difficulties in focusing on the task-relevant information as they become distracted by salient features within the environment. This is in line with the attention inhibition deficit in ageing reported in past studies (e.g., Hasher \& Zacks, 1988). According to this account, older adults exhibit top-down control difficulties, with attention orienting being more affected by stimulus properties rather than the task at hand (Olk \& Kingstone, 2015; West, 1996). Lastly, older adults may have difficulties in selecting the appropriate information required to solve the task. This is consistent with our findings that older adults have difficulties in extracting useful information from the stimuli (Segen et al., 2020).

In our earlier study (Segen et al., 2020), we could not distinguish between these explanations for several reasons. First, we did not systematically manipulate the availability of landmarks. Second, the landmarks used in the environment were all unique and informative and could, therefore, facilitate the encoding of the object locations, even if they distracted the older participants. Third, there was substantial overlap between the landmarks and the objects of the scene, which prevented a region of interest analysis. Finally, due to the large perspective shifts introduced in some trials (e.g. $135^{\circ}$ ), some landmarks were visible during encoding but not at test.

The current study was designed to disentangle the explanations for age-related differences in place recognition by examining gaze behaviour. To do so, we amended our original task (Segen et al., 2020) in a variety of ways to overcome the limitations of the earlier study. First, we reduced the size of the perspective shift between encoding and test which allowed us to present the same landmarks during learning and test, ensuring that participants could use the information they encoded during learning to solve the task at test. Decreasing the size of the perspective shift also made the task easier (Hegarty \& Waller, 2004; Montofinese et al., 2015; Segen et al., 2020; Muffato et al., 2019; Hilton et al., 2020). Task difficulty was further reduced by including only the condition in which two object clusters were swapped with each other. Reducing task difficulty aimed at avoiding floor level performance in older adults, which would allow us to rule out that potential differences in gaze behaviour across groups are caused by participants' inability to carry out the task.

Generally, we predict a decline in performance in older adults consistent with age-related place recognition deficits (Hartley et al., 2007). Responding after a perspective shift requires additional and demanding mental manipulations of the stored representations (e.g., mentally rotating the new or the stored representation to match the other, imagining moving around the array; Hegarty \& Waller, 2004; Holmes et al., 2018; King et al., 2002). Therefore, we expect that the introduction of the perspective shift would impair performance in both groups. However, we predict a larger decrease in performance in older adults who seem to have difficulties with initiating those mental manipulations as reflected in past findings documenting larger impairments with the introduction rather than the increase of the perspective shift (Montofinesse et al., 2015; Muffato et al., 2019; Hilton et al., 2020; Segen et al., 2020).

To investigate the role of landmarks in encoding strategies and performance, we included trials in which landmarks (in the form of posters on the walls) were: (1) unique and could be used to encode object locations, (2) identical and thus uninformative or (3) absent from the scene. Varying the availability and utility of room-based landmarks allowed us to test whether age-related differences in gaze behaviour during spatial encoding were due to older adults encoding object positions by relating them 
to the landmarks or to older adults having difficulties in selecting and/or focusing on task-relevant information.

Since this part of the study is largely exploratory, we have formulated a series of predictions about results that we would expect to find depending on how older adults use additional landmarks during encoding of object locations. Given that the task can be solved either by focusing on the local arrangement of objects or by relating object positions to landmarks, we should not necessarily expect age-related differences in performance if older adults simply shift towards a particular encoding strategy depending on which information is available. However, if older adults select an encoding strategy that depends on the availability of landmarks as suggested by our previous research (Segen et al., 2020), we expect them to perform better when landmarks are informative than uninformative. Finally, if older adults have difficulties focusing on task-relevant information as a result of an attention inhibition deficit (Hasher \& Zacks, 1988), and are therefore distracted by the presence of landmarks, we predict worse performance when landmarks are available (either informative or uninformative) than when they are not.

In terms of gaze behaviour, if older adults rely more on landmarks as part of their encoding strategy, compared to their younger counterparts, we expect them to spend more time gazing at informative landmarks than uninformative landmarks. If, however, older adults are distracted by the landmarks, we expected them to show similar gaze behaviour in conditions with informative and uninformative landmarks.

\section{Method}

\section{Participants}

Twenty-eight young (mean age $=21.00$ years, $\mathrm{SD}=2.27$; age range $=18-27$ years; 15 females and 13 males) and 32 older adults aged 60 years and over (mean age $=68.80, \mathrm{SD}=6.34$, age range $=60-85 ; 17$ females and 15 males) took part in this study. Participants were recruited either through the participant recruitment system of Bournemouth University or through opportunity sampling in the community. Older adults received monetary compensation for their time whilst younger participants received course credits. Participants were screened for mild cognitive impairment using the Montreal Cognitive Assessment (MoCA; Nasreddine et al., 2005). Based on a threshold score of 23 , no participants were excluded (Luis et al., 2009; Waldron-Perrine \& Axelrod, 2012). All participants gave their written informed consent in accordance with the Declaration of Helsinki (World Medical Association 2013).

\section{Virtual environment}

The virtual environment was designed with Adobe 3DS Max 2018 and depicted a $13.5 \mathrm{~m} \times 14.6 \mathrm{~m}$ rectangular room. The room contained 6 identical objects; pink vases on metal stands that were arranged in three clusters of 1,2 and 3 objects in the centre of the room (see Fig. 1). In the No Landmarks condition, the walls contained no additional cues, in the Uninformative Landmarks condition eight identical posters of the Tower Bridge were presented, two on each wall. Finally, in the Informative Landmarks condition eight unique posters were presented, again two on each wall. These posters consisted of highly familiar and recognisable landmarks (Hamburger \& Röser, 2014): the Leaning Tower of Pisa, Stonehenge, the Statue of Liberty, the Golden Gate Bridge, the Eiffel Tower, the White House, the Big Ben, and the Great Wall of China.

The experimental stimuli were renderings of the environment with a $70^{\circ}$ horizontal field of view (FOV) with a $15 \%$ downward shift in the vertical FOV, yielding an asymmetric viewing frustum to simulate human vision. The virtual cameras from which the static images of the scenes were rendered were arranged on a circle (radius of $6.7 \mathrm{~m}$ ) at $30^{\circ}$ intervals, providing 12 possible camera positions and the object clusters were arranged in six unique layouts within the room (Fig. 1b-d). Six of those camera positions were used in the learning phase and in the $0^{\circ}$ perspective shift condition. The remaining 6 viewpoints were used in the test phase in the $30^{\circ}$ perspective shift condition. Stimuli were presented as static images on a desktop computer with OpenSesame 3.1.7 (Mathôt et al., 2012) and a standard computer keyboard was used to collect responses.

\section{Eye-tracking}

Eye movements were recorded using an Eyelink II (SR Research) head-mounted eye tracker at a rate of $500 \mathrm{~Hz}$. Calibrations were performed at least three times and drift correction was performed before each trial. The experiment was presented on a $102 \mathrm{~cm}$ screen (diagonal) with an aspect ratio of $16: 9$ and a resolution of $1920 \times 1080$ pixels. Participants were seated $100 \mathrm{~cm}$ from the monitor resulting in a physical horizontal FOV of $47.9^{\circ}$ and $28^{\circ}$ vertical FOV.

\section{Procedure}

Each experimental trial started with a fixation cross and a scrambled stimuli mask presented for $1500 \mathrm{~ms}$ (Fig. 1a). In the learning phase, participants were presented with a rendering of one of the 6 unique configurations of the target objects from one of the six possible viewpoints for $12 \mathrm{~s}$. After this learning phase, participants were again presented with a fixation cross and a scrambled stimuli mask for 
A

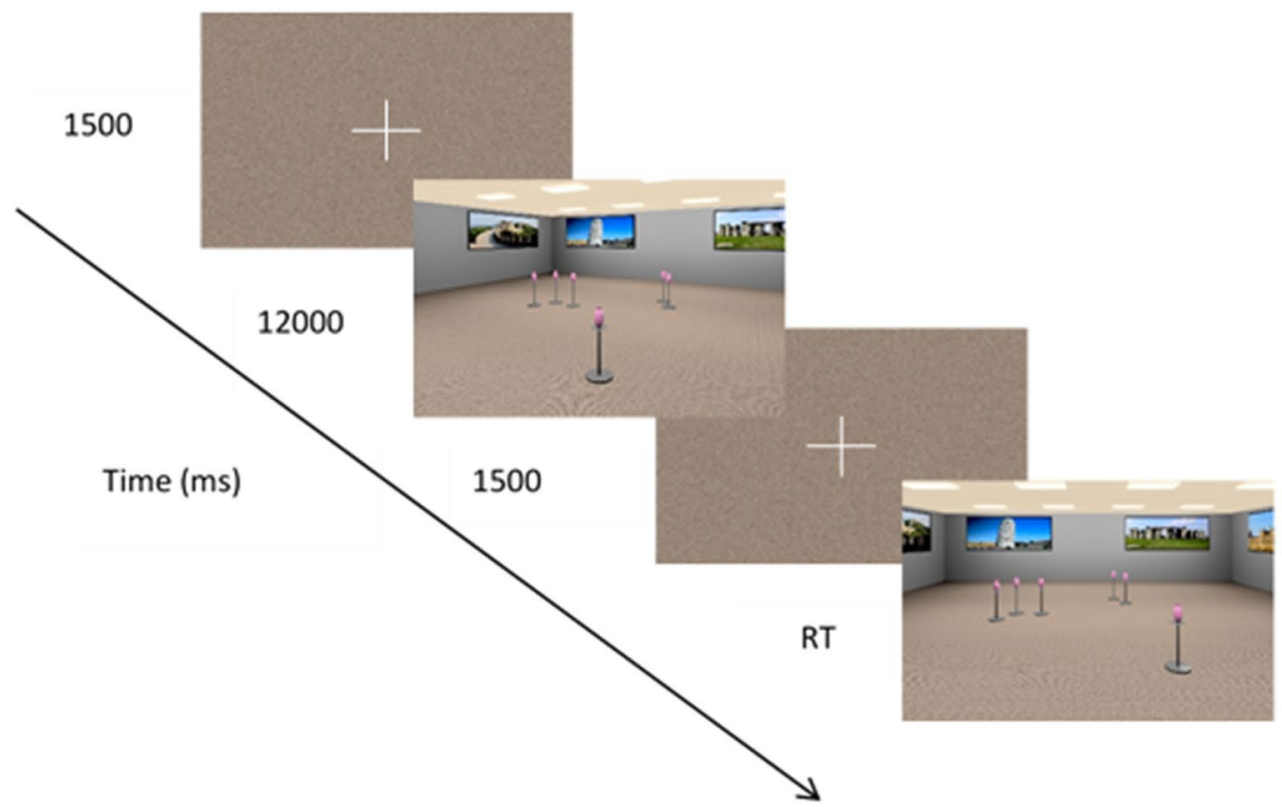

\section{B No Cues + Swap $+30^{\circ}$ Perspective Shift}
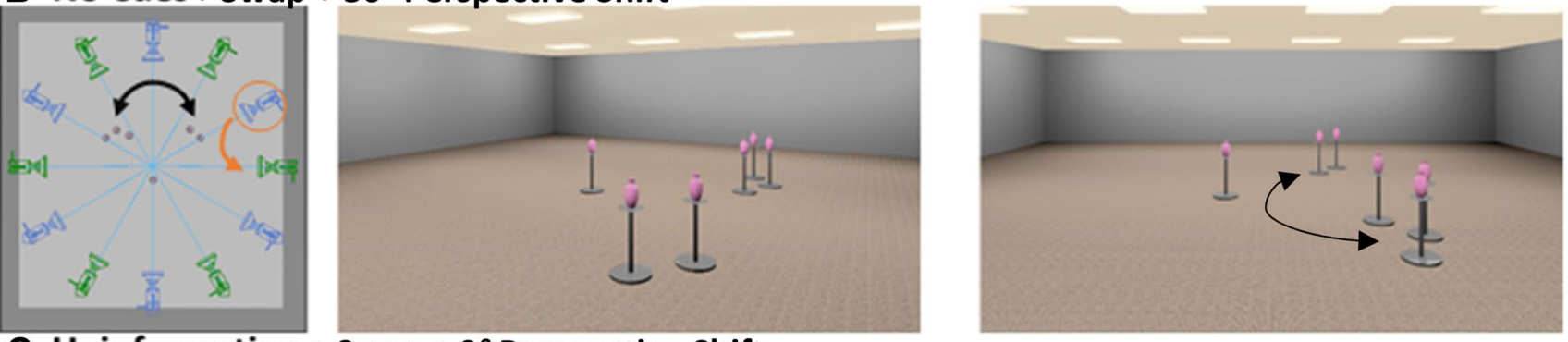

C Uninformative + Swap $+0^{\circ}$ Perspective Shift
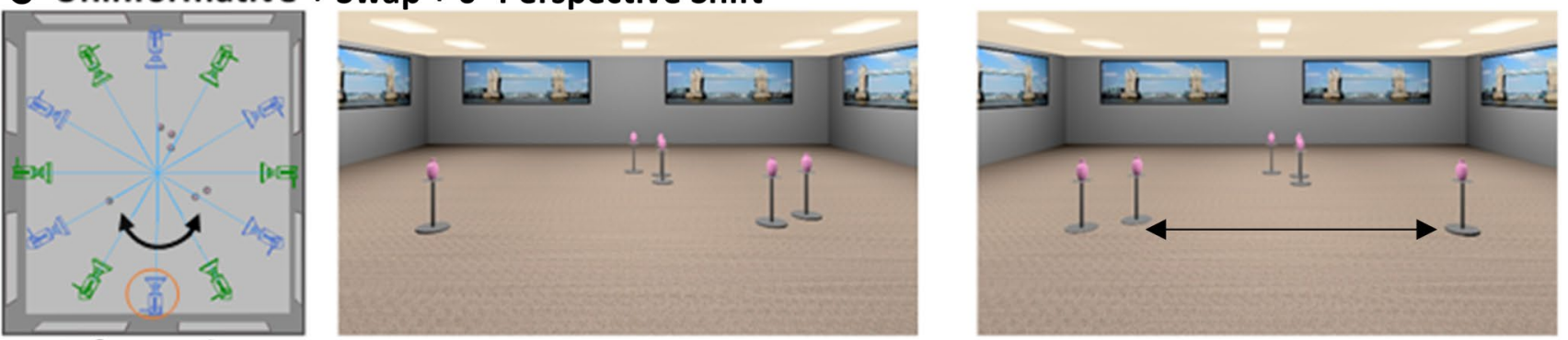

\section{Informative + Same \& $30^{\circ}$ Perspective Shift}
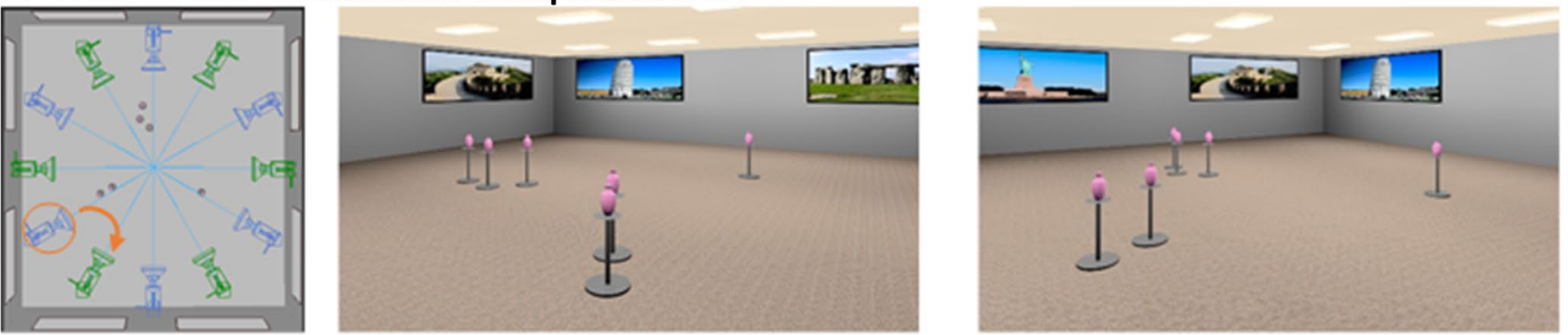

Fig. 1 a Experimental protocol; b, c and d Virtual environment and stimuli for the experimental task, Blue and Green cameras represent the possible virtual cameras positions for the Learning and Test phase, respectively. Examples of possible object cluster layouts are shown in b (No Landmarks), c (Uninformative Landmarks) and d (Informative Landmarks). The left panel shows a survey perspective of the example trials, indicating the rotation of the camera (Orange arrow) and swapping of the two object clusters (Black arrow) in Swap trials (b, c). The middle and right panels show the two corresponding snapshots for the learning and test phases, respectively. In $\mathbf{b}$ and $\mathbf{d}$ there is a $30^{\circ}$ perspective shift, to the left and right respectively. In $\mathbf{c}$ there is no perspective shift. The black arrows in the right panel $(\mathbf{b}, \mathbf{c})$ indicate which clusters were swapped on the test stimuli 
$1500 \mathrm{~ms}$. Then, in the test phase they were presented with a rendering of the room either from the same viewpoint $(50 \%$ of trials, Fig. 1c) or a different viewpoint that was offset by $30^{\circ}$ from the study viewpoint (Fig. $1 \mathrm{~b}$, d). Participants were asked to respond by pressing the $\mathrm{x}$ or $\mathrm{m}$ keys on the keyboard as to whether the target objects were in the same locations as during the training phase or not. In $50 \%$ of the trials, the target objects remained in the same locations (Same, Fig. 1c) and in the other $50 \%$ of the trials, two of the three object clusters swapped locations (Swap, Fig. 1b, c). As a result, chance level performance for this task was $50 \%$.

The experiment consisted of 144 experimental trials that were preceded by 6 practice trials. The entire study took around $90 \mathrm{~min}$ to complete and participants were allowed to take breaks when they wished.

\section{Design}

The experiment followed a mixed 2 (Age Group: young vs. older adults) $\times 2$ (Manipulation: Same, Swap, Fig. 1b,c and d) $\times 2$ (Perspective Shift: $\left.0^{\circ}, 30^{\circ}\right) \times 3$ (Landmark Type: $N o$ Landmarks, Uninformative, Informative) design with Manipulation, Perspective Shift and Landmark Type manipulated within participants and Age Group between.

\section{Data Analysis}

Data from one older participant were excluded from all analyses due to chance level performance in the $0^{\circ}$ Perspective Shift condition. The remaining data were analysed with linear mixed-effects models (LME) using LME4 (Bates et al., 2018) in R (R Core Team, 2013). Specifically, accuracy was analysed using generalized linear mixed-effects (GLME) models with the glmer function from LME4 package. The following contrasts were used in all (G)LMEs conducted: Age Group (Younger adults/Older adults), Perspective shift $\left(0^{\circ} / 30^{\circ}\right)$ and Manipulation (No Change/Swap) were coded using effect coding. This coding scheme compares the effect of a variable (i.e. Age Group) on performance averaged across all levels of other variables (i.e. Perspective Shift and Manipulation). Landmark Type was coded using treatment coding. Since we were interested in examining the difference between Informative and Uninformative Landmarks and the difference between No Landmarks and Uninformative Landmarks, we used the Uninfomative Landmark as the baseline. As a result, all of the effects for other factors are calculated with reference to the performance in the Uninformative Landmark, rather than the average of performance for all levels of Landmark Type. For the response time analysis, we included only the correct trials and we logtransformed response times following the recommendations of Baayen et al. (2008) for dealing with the skewness of the response time distribution. Prior to transforming, response times below $200 \mathrm{~ms}$ and over 20,000 ms were removed.

\section{Results}

\section{Accuracy}

Accuracy estimates were obtained for each participant with Age Group, Perspective Shift, Landmark Type and Manipulation as fixed factors and a random by-subject and by-item intercept. Coefficients, standard errors and $z$-values (Table 1) indicate that Perspective Shift and Manipulation affected performance. Specifically, accuracy decreased with the introduction of a $30^{\circ}$ Perspective Shift (Fig. 2a) and in the Swap condition (Fig. 2b). In addition, there was an interaction between Perspective Shift and Manipulation with a greater decline in performance in the No Change condition compared to the Swap condition following a $30^{\circ}$ Perspective Shift (Fig. 2c). Finally, we found a three-way interaction between Perspective Shift, Manipulation and Age Group with older adults showing a larger decline in performance than younger adults in the No Change condition when a $30^{\circ}$ Perspective Shift was introduced, whilst displaying an increase in performance in the Swap condition when a $30^{\circ}$ Perspective Shift was introduced (Fig. 2d). Effect plots for significant main effects and interactions are reported in the Supplementary Materials.

\section{Response Time}

As with accuracy, response time estimates were obtained for each participant with Age Group, Perspective Shift, Landmark Type and Manipulation as fixed factors and a random by-subject and by-item intercept with a random slope for Manipulation across participants. Coefficients, standard errors and $t$-values (Table 2) show that Age Group, Perspective Shift, Landmark Type and Manipulation were all reliable predictors of response time. Specifically, we found that older adults were slower to respond compared to younger adults (Fig. 3a), and that response times increased with the introduction of a Perspective Shift (Fig. 3b). In addition, response times were longer with Informative than Uninformative Landmark Type (Fig. 3c) and in the Swap condition compared to the No Change condition (Fig. 3d). We also found a significant interaction between Age Group and Manipulation with a smaller increase in response times in the Swap condition in older than younger adults (Fig. 3e). There was also a Perspective Shift and Manipulation interaction with a smaller increase in response times in the Swap condition than the No Change condition with the introduction of the Perspective Shift (Fig. 3f). We also found an interaction between Landmark Type and Manipulation with a smaller increase in response times between the $\mathrm{No}$ 
Table 1 Coefficients from Accuracy GLME analysis

\begin{tabular}{|c|c|c|c|}
\hline \multirow[t]{2}{*}{ Predictors } & \multicolumn{3}{|l|}{ Accuracy } \\
\hline & Coefficients & Std. Error & $z$-value \\
\hline (Intercept) & 2.023 & 0.262 & 7.724 \\
\hline Age Group $($ Old $)$ & 0.145 & 0.112 & 1.293 \\
\hline Perspective Shift $\left(30^{\circ}\right)$ & -0.635 & 0.079 & -8.049 \\
\hline Landmark Type (Informative) & -0.122 & 0.347 & -0.350 \\
\hline Landmark Type (No Landmarks) & 0.066 & 0.350 & 0.189 \\
\hline Manipulation (Swap) & -1.316 & 0.086 & -15.216 \\
\hline Age Group $($ Old $)$ : Perspective Shift $\left(30^{\circ}\right)$ & -0.104 & 0.071 & -1.468 \\
\hline Age Group (Old): Landmark Type (Informative) & -0.063 & 0.095 & -0.659 \\
\hline Age Group $($ Old $)$ : Landmark Type (No Landmarks) & -0.138 & 0.105 & -1.314 \\
\hline Perspective Shift $\left(30^{\circ}\right)$ : Landmark Type (Informative) & 0.176 & 0.106 & 1.659 \\
\hline Perspective Shift $\left(30^{\circ}\right)$ : Landmark Type (No Landmarks) & -0.037 & 0.116 & -0.319 \\
\hline Age Group $(O l d)$ : Manipulation (Swap) & 0.063 & 0.071 & 0.887 \\
\hline Perspective Shift $\left(30^{\circ}\right)$ : Manipulation (Swap) & 0.414 & 0.077 & 5.387 \\
\hline Landmark Type (Informative): Manipulation (Swap) & 0.212 & 0.115 & 1.846 \\
\hline Landmark Type (No Landmarks): Manipulation (Swap) & -0.082 & 0.125 & -0.656 \\
\hline Age Group $(O l d)$ : Perspective Shift $\left(30^{\circ}\right)$ : Landmark Type (Informative) & 0.097 & 0.095 & 1.020 \\
\hline Age Group $($ Old $)$ : Perspective Shift $\left(30^{\circ}\right)$ : Landmark Type (No Landmarks) & 0.137 & 0.105 & 1.303 \\
\hline Age Group $($ Old $)$ : Perspective Shift $\left(30^{\circ}\right)$ : Manipulation (Swap) & 0.240 & 0.071 & 3.399 \\
\hline Age Group (Old): Landmark Type (Informative): Manipulation (Swap) & 0.049 & 0.095 & 0.514 \\
\hline Age Group (Old): Landmark Type (No Landmarks): Manipulation (Swap) & 0.054 & 0.105 & 0.512 \\
\hline Perspective Shift $\left(30^{\circ}\right)$ : Landmark Type (Informative): Manipulation (Swap) & 0.060 & 0.103 & 0.584 \\
\hline Perspective Shift $\left(30^{\circ}\right)$ : Landmark Type (No Landmarks): Manipulation (Swap) & 0.155 & 0.114 & 1.364 \\
\hline Age Group $(\mathrm{Old})$ : Perspective Shift $\left(30^{\circ}\right)$ : Landmark Type (Informative): Manipulation (Swap) & -0.122 & 0.095 & -1.277 \\
\hline Age Group $($ Old $)$ : Perspective Shift $\left(30^{\circ}\right)$ : Landmark Type (No Landmarks): Manipulation (Swap) & -0.201 & 0.105 & -1.916 \\
\hline
\end{tabular}

Significant $z$ values $(|z| \geq 1.96)$ in bold

Fig. 2 Bar plots of accuracy values for a significant main effect of a Perspective Shift, b Manipulation, and significant interactions $\mathbf{c}$ between Manipulation and Perspective Shift and $\mathbf{d}$ Interaction between Age Group, Manipulation and Perspective Shift with a mean (solid line) and 95\% CIs (grey shaded area) with individual data points and violin plots behind
A

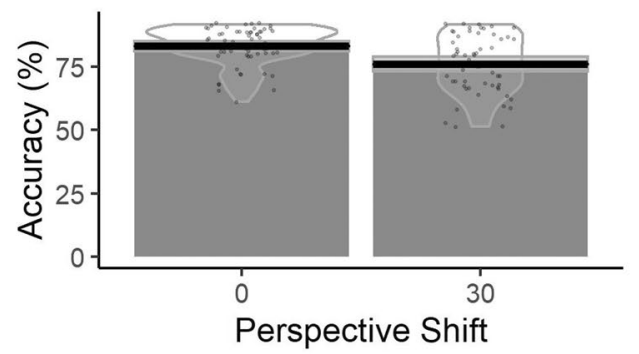

C

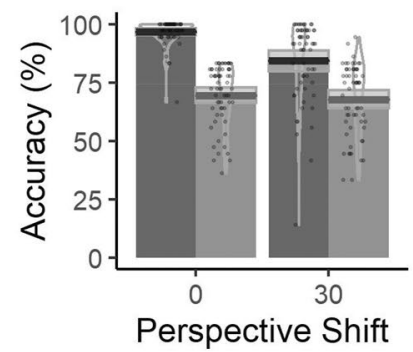

B

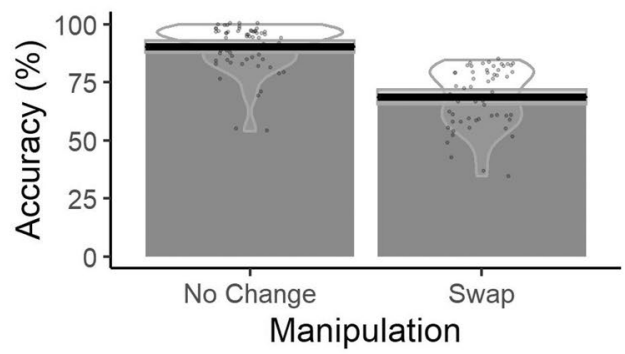

D

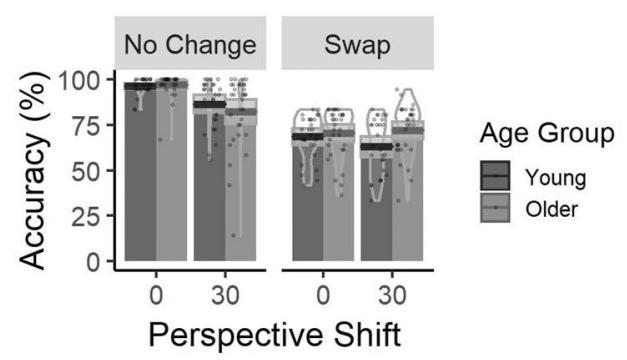


Table 2 Coefficients from response time LME analysis

\begin{tabular}{|c|c|c|c|}
\hline \multirow[t]{2}{*}{ Predictors } & \multicolumn{3}{|c|}{ Log transformed response time } \\
\hline & Estimates & Std. Error & $t$-value \\
\hline (Intercept) & 7.834 & 0.041 & 190.067 \\
\hline Age Group & 0.209 & 0.040 & 5.248 \\
\hline Perspective Shift $\left(30^{\circ}\right)$ & 0.130 & 0.015 & 8.459 \\
\hline Landmark Type (Informative) & 0.058 & 0.020 & 2.942 \\
\hline Landmark Type (No Landmarks) & -0.013 & 0.020 & -0.640 \\
\hline Manipulation (Swap) & 0.133 & 0.011 & 12.386 \\
\hline Age Group: Perspective Shift $\left(30^{\circ}\right)$ & 0.006 & 0.014 & 0.451 \\
\hline Age Group: Landmark Type (Informative) & 0.019 & 0.013 & 1.470 \\
\hline Age Group: Landmark Type (No Landmarks) & -0.019 & 0.013 & -1.443 \\
\hline Perspective Shift $\left(30^{\circ}\right)$ : Landmark Type (Informative) & 0.012 & 0.015 & 0.813 \\
\hline Perspective Shift $\left(30^{\circ}\right)$ : Landmark Type (No Landmarks) & -0.000 & 0.015 & -0.007 \\
\hline Age Group: Manipulation (Swap) & -0.032 & 0.009 & -3.474 \\
\hline Perspective Shift $\left(30^{\circ}\right)$ : Manipulation (Swap) & -0.077 & 0.010 & -7.542 \\
\hline Landmark Type (Informative): Manipulation (Swap) & -0.034 & 0.015 & -2.259 \\
\hline Landmark Type (No Landmarks): Manipulation (Swap) & 0.010 & 0.015 & 0.654 \\
\hline Age Group: Perspective Shift (30): Landmark Type (Informative) & -0.003 & 0.013 & -0.239 \\
\hline Age Group: Perspective Shift $\left(30^{\circ}\right)$ : Landmark Type (No Landmarks) & -0.013 & 0.013 & -1.012 \\
\hline Age Group: Perspective Shift $\left(30^{\circ}\right)$ : Manipulation (Swap) & -0.018 & 0.009 & -1.960 \\
\hline Age Group: Landmark Type (Informative): Manipulation (Swap) & -0.008 & 0.013 & -0.596 \\
\hline Age Age Group: Landmark Type (No Landmarks): Manipulation (Swap) & -0.024 & 0.013 & -1.847 \\
\hline Perspective Shift $\left(30^{\circ}\right)$ : Landmark Type (Informative): Manipulation (Swap) & 0.019 & 0.014 & 1.312 \\
\hline Perspective Shift $\left(30^{\circ}\right)$ : Landmark Type (No Landmarks): Manipulation (Swap) & 0.002 & 0.014 & 0.162 \\
\hline Age Group: Perspective Shift $\left(30^{\circ}\right)$ : Landmark Type (Informative): Manipulation (Swap) & 0.005 & 0.013 & 0.406 \\
\hline Age Group: Perspective Shift $\left(30^{\circ}\right)$ : Landmark Type (No Landmarks): Manipulation (Swap) & -0.004 & 0.013 & -0.289 \\
\hline
\end{tabular}

Significant $t$ values $(|t| \geq 1.96)$ in bold

Change and the Swap condition in the Informative Landmark Type (Fig. 3g) compared to Uninformative Landmark Type condition. Finally, we found a three-way interaction between Age Group, Perspective Shift and Manipulation, with the Age Group and Perspective Shift interactions showing a different trend across No Change and Swap Manipulation. Specifically, there was a larger increase in response times in older adults than young adults, in the No Change condition with the introduction of the Perspective Shift (Fig. 3). Whilst in the Swap condition, the increase in response times in older adults was smaller when a Perspective Shift was introduced compared to young adults. Effect plots for significant main effects and interactions are reported in the Supplementary Materials.

\section{Response Bias}

To examine if participants displayed a response bias, we carried out an analysis based on Signal Detection Theory (Harvey, 1992; Macmillan \& Creelman, 1991) using the sdt.rmcs (Todorova, 2017) package in R. Signal Detection
Theory evaluates sensitivity and response bias in situations that require decision making under uncertainty. It is applied when a binary decision about the presence or absence of a signal is made, comparing the response with the actual presence/absence of the signal. With Signal Detection Theory, the formula $\mathrm{c}=-0.5[\mathrm{z}$ (hit rate $)+z$ (false alarm rate) is used to compute response bias, where hit rate and false alarm rates refer to trials in which the signal was correctly or incorrectly, respectively, reported as present.

Overall, there was a positive response bias showing that participants were more likely to respond that nothing has changed than to respond that something had changed (Fig. 4). LMM analysis (Table 3) with Age Group, Perspective Shift and Landmark Type as fixed factors and bysubject intercept with a random slope for Perspective Shift, indicated that the introduction of a Perspective Shift led to a decrease in response bias, which was larger in older adults than in younger adults. Furthermore, when a Perspective Shift was introduced, the response bias decreased more in the No Landmarks and the Informative Landmarks 

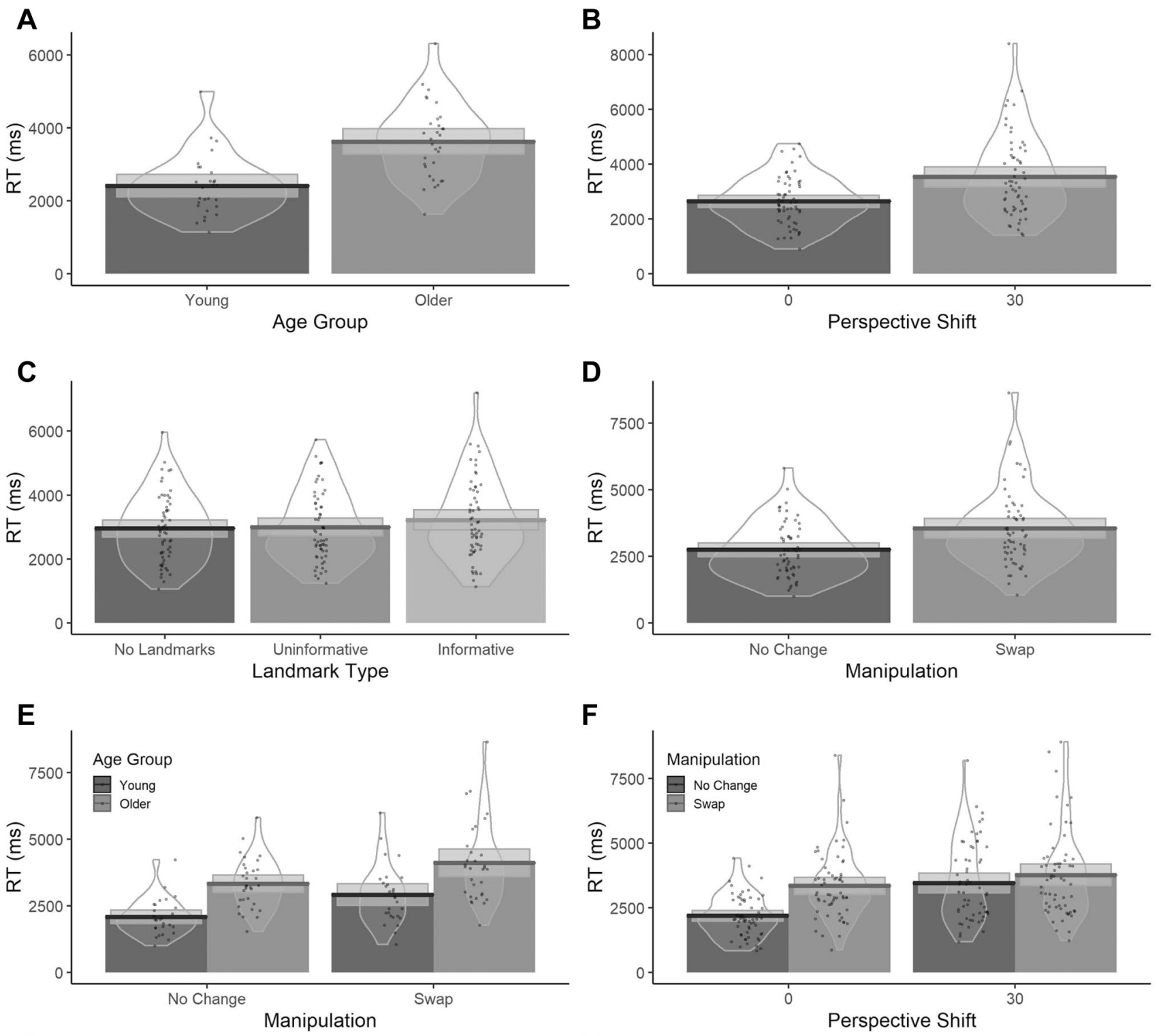

\section{G}

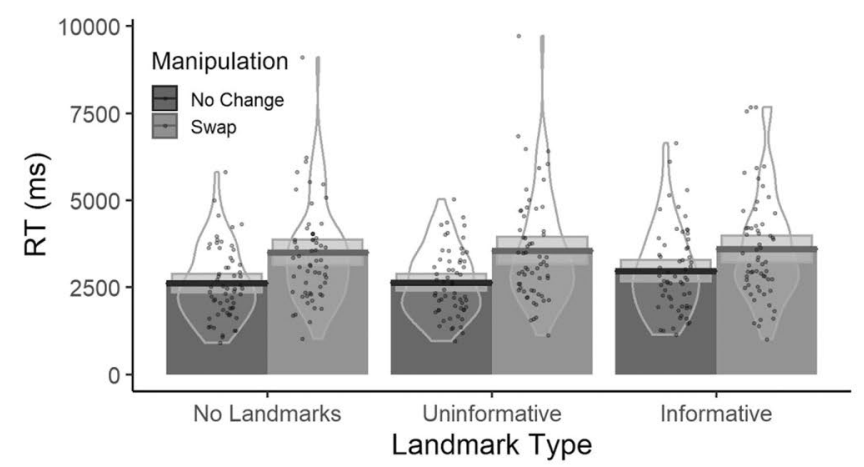

Fig. 3 Bar plots of Response Times values for significant main effects of A: Age Group B: Perspective Shift C: Landmark Type (significant only for the Informative Landmark Type) D: Manipulation and interactions between E: Age Group and Manipulation F: Perspective Shift and Manipulation G: Landmark Type and Condition (significant
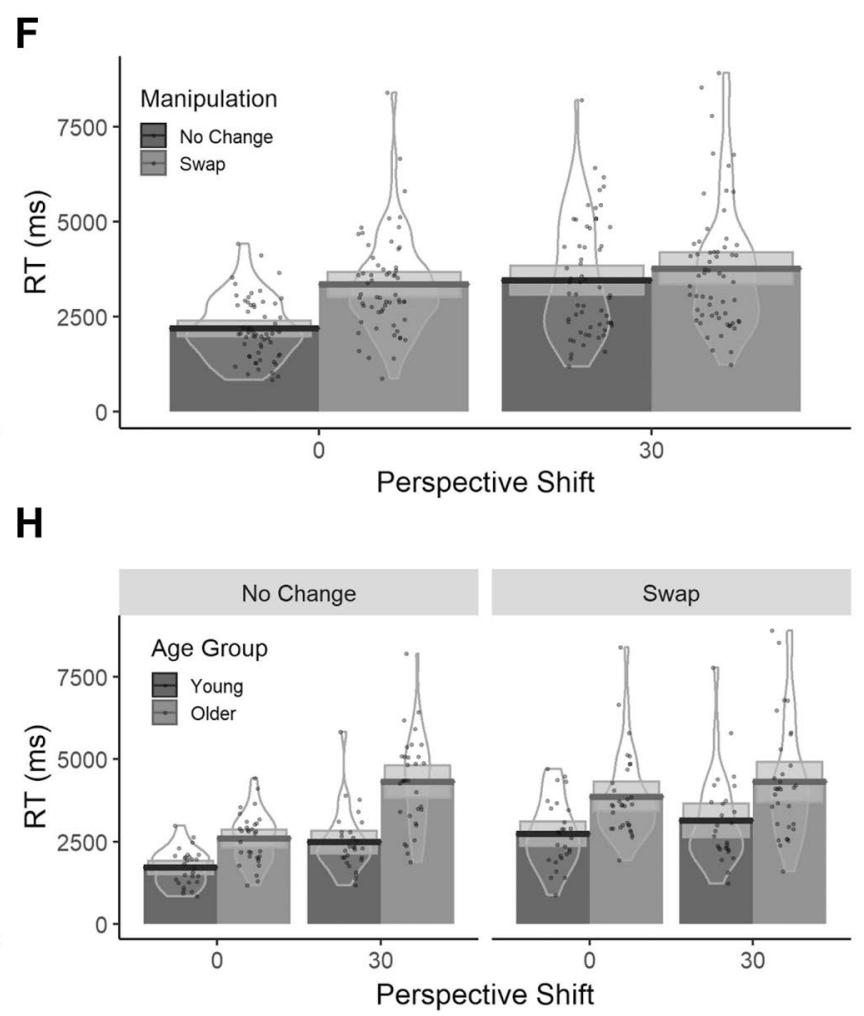

only for Landmark Type (Informative): Manipulation (Swap)) H: Age Group, Manipulation and Perspective Shift with mean (solid line) and 95\% CIs (grey shaded area) with individual data points and violin plots behind 
Fig. 4 Bar plots for Response Bias as a function of Age Group, Landmark Type and Perspective Shift with mean (solid line) and 95\% CIs (grey shaded area) with individual data points and violin plots behind. Stars indicate response bias significantly different from 0 (1 star $[p<0.05], 2$ stars $[p<0.01]$ and 3 stars $[p<0.001])$

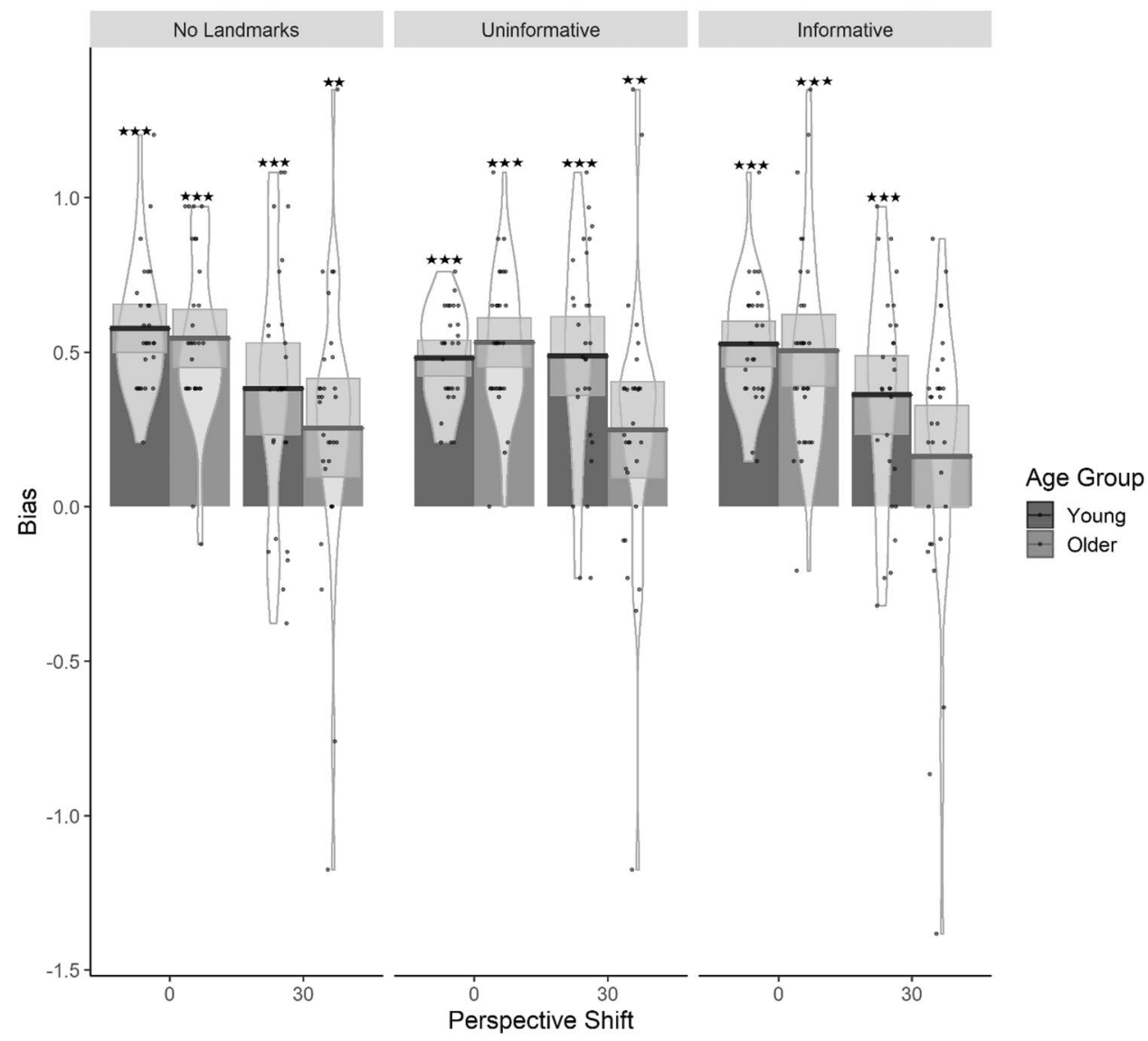

Table 3 Coefficients from Response Bias LME analysis

\begin{tabular}{|c|c|c|c|}
\hline \multirow[t]{2}{*}{ Predictors } & \multicolumn{3}{|c|}{ Response bias (c) } \\
\hline & Estimates & Std. Error & $t$-value \\
\hline (Intercept) & 0.437 & 0.033 & 13.043 \\
\hline Age Group (Older Adults) & -0.047 & 0.033 & -1.403 \\
\hline Perspective Shift $\left(30^{\circ}\right)$ & -0.069 & 0.029 & -2.384 \\
\hline Landmark Type (Informative) & 0.003 & 0.026 & 0.097 \\
\hline Landmark Type (No Landmarks) & -0.048 & 0.026 & -1.826 \\
\hline Age Group: Perspective Shift $\left(30^{\circ}\right)$ & -0.072 & 0.029 & -2.495 \\
\hline Age Group: Landmark Type (Informative) & 0.007 & 0.026 & 0.264 \\
\hline Age Group: Landmark Type (No Landmarks) & -0.008 & 0.026 & -0.306 \\
\hline Perspective Shift $\left(30^{\circ}\right)$ : Landmark Type (Informative) & -0.052 & 0.026 & -1.978 \\
\hline Perspective Shift $\left(30^{\circ}\right)$ : Landmark Type (No Landmarks) & -0.058 & 0.026 & -2.201 \\
\hline Age Group: Perspective Shift $\left(30^{\circ}\right)$ : Landmark Type (Informative) & 0.049 & 0.026 & 1.845 \\
\hline Age Group: Perspective Shift $\left(30^{\circ}\right)$ : Landmark Type (No Landmarks) & 0.028 & 0.026 & 1.043 \\
\hline
\end{tabular}

Significant $t$ values $(|t| \geq 1.96)$ in bold

conditions compared to the Uninformative Landmarks con- Gaze analysis

dition (Table 4).
Fixations and saccades were identified using the SR Research algorithms and were pre-processed as follows: First, we removed fixations that contained a blink, fell 
Table 4 Coefficients from Dwell Time on the top IA LME analysis

\begin{tabular}{lccr}
\hline Predictors & \multicolumn{3}{c}{ Dwell Time on Landmarks } \\
\cline { 2 - 4 } & Estimates & Std. Error & t-value \\
\hline (Intercept) & 13.054 & 1.503 & 8.684 \\
Age Group (Older Adults) & $2.99 /$ & 1.457 & $\mathbf{2 . 0 5 8}$ \\
Landmark Type (No Land- & -8.108 & 0.644 & $\mathbf{- 1 2 . 6 0 0}$ \\
$\quad$ marks) & & & \\
Landmark Type (Informative) & 9.540 & 0.644 & $\mathbf{1 4 . 8 2 6}$ \\
Age Group (Older Adults): & -1.804 & 0.375 & $\mathbf{- 4 . 8 1 2}$ \\
$\quad$ Landmark Type (No Land- \\
$\quad$ marks)
\end{tabular}

Significant $t$ values $(|t| \geq 1.96)$ in bold

outside of the screen boundaries or were shorter than $80 \mathrm{~ms}$ or longer than 1000 ms (Inhoff \& Radach, 1998; Nuthmann, 2017). Finally, we removed saccades with maximum amplitudes $\left(41.35^{\circ} \mathrm{va}\right)$ or velocities $\left(1500^{\circ} / \mathrm{s}\right)$ larger than it should be possible based on the distance of the participant from the screen and the screen size.

The primary aim of the gaze analysis was to investigate age differences in encoding strategies and was therefore mainly focused on the analysis of gaze during the encoding phase. Analysis of differences in basic saccade and fixation parameters between young and older adults showed that during the $12 \mathrm{~s}$ encoding period, older adults made shorter and more frequent fixations as well as more frequent saccades. The results are reported in detail in the supplementary materials.

\section{Gaze on landmarks}

As we were primarily interested in age-related differences in gaze as a function of Landmark Type, we split stimuli into two interest areas (See Fig. 5) and compared the percentage of Dwell Time on the top interest area (IA) where Landmarks were located when available vs. the bottom area where the objects were located. To do so, we computed the total dwell time for each trial by adding up the duration of all fixations in the trial. Next, we calculated the proportion of dwell time that was spent fixating in the top IA. This approach allowed us to specifically focus on age-related differences in the use of room-based Landmarks during encoding with the increased Dwell Time on the upper part of the stimuli largely reflecting gaze on Landmarks (when available).

LME analysis with Age Group and Landmark Type as fixed factors and a by-subject and by-item random intercept showed that Landmark Type and Age Group were reliable predictors of Dwell Time on the top IA. Specifically, we found that compared to the Uninformative Landmarks condition that was used as a baseline, there was a reduction in Dwell Time on the top IA in the No Landmarks and an increase in Dwell Time in the Informative Landmarks condition. We also found that older adults spent more time looking at the top IA compared to younger adults. In addition, there was a Landmark Type and Age Group interaction whereby older adults' Dwell Time on Landmarks decreased more than that of younger adults' in the No Landmarks condition compared to Uninformative Landmarks condition and showed a larger increase in the Informative Landmarks condition compared to the Uninformative Landmarks condition. A Dwell Time analysis on the top IA at test produced similar results to those of the learning phase, with the exception that the increase in Dwell Time in older adults and the Age Group by Landmark Type (No Landmarks) interaction were not significant. Results from this analysis are presented in the Supplementary Materials.

\section{Relationship between Gaze and Performance}

Dwell time on the top IA was not related to performance across any of the three Landmark Type conditions (Fig. 6), suggesting that the task could be solved either by using Landmarks (when they are available) or by focusing primarily on the objects. Thus, the differences in gaze behaviour reported here are likely to represent differences in encoding strategy preferences that change with age.

\section{Gaze behaviour across trials}

We also investigated if gaze behaviour changes across time by correlating Dwell Time on landmarks with trial older for younger and older participants in the No Landmark, Uninformative and Informative Landmark conditions. We found that across both younger and older adults, Dwell Time remained consistent in the No Landmark condition throughout the experiment (Young: $r=0.011, p=0.895$, Older: $r=-0.09, p=0.279)$. In the Uninformative Landmark condition, older adults spent less time fixating on landmarks over the course of the experiment $(r=-0.18, p=0.032)$, whilst younger adults' gaze $(r=-0.05, p=0.543)$ remained unchanged. In the Informative Landmark condition, an opposite pattern of results was found with younger adults spending less time fixating on landmarks $(r=-0.20, p=0.018)$ with older adults' gaze remaining unchanged $(r=-0.09$, $p=0.266$ ).

\section{Consistency in gaze between learning and test}

Finally, we examined if participants showed similar gaze behaviour during learning and test. To do so, we correlated the 
Fig. 5 Heatmaps representing number of fixations as a function of age group and landmark type

\section{No Landmarks}

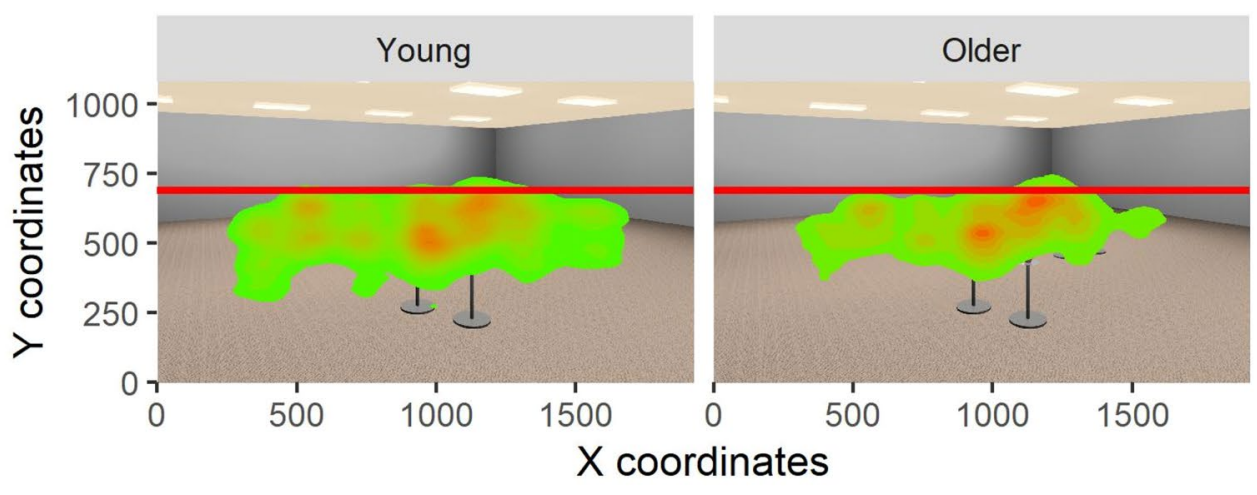

Uninformative Landmarks

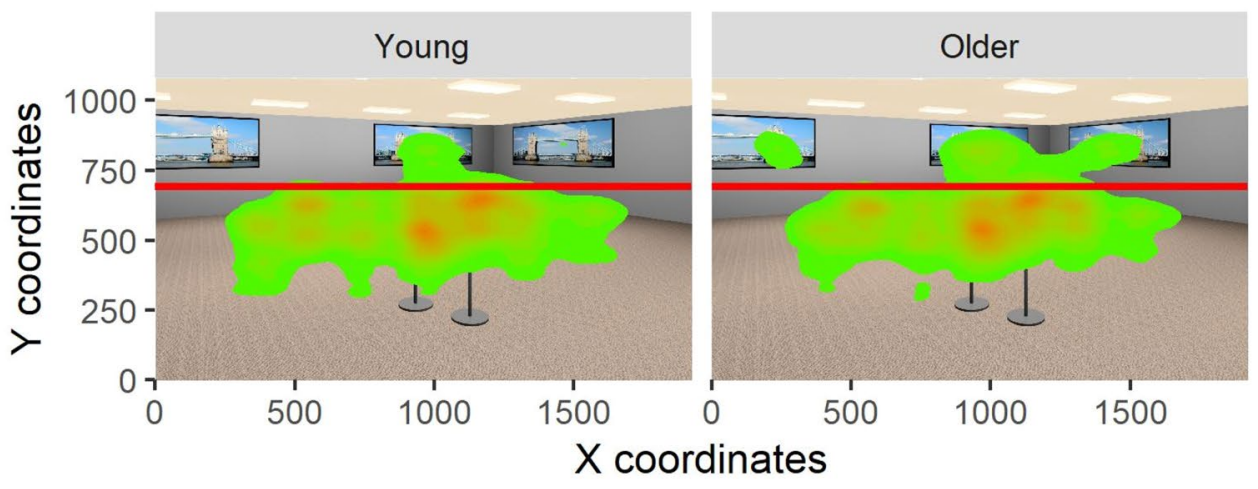

Informative Landmarks

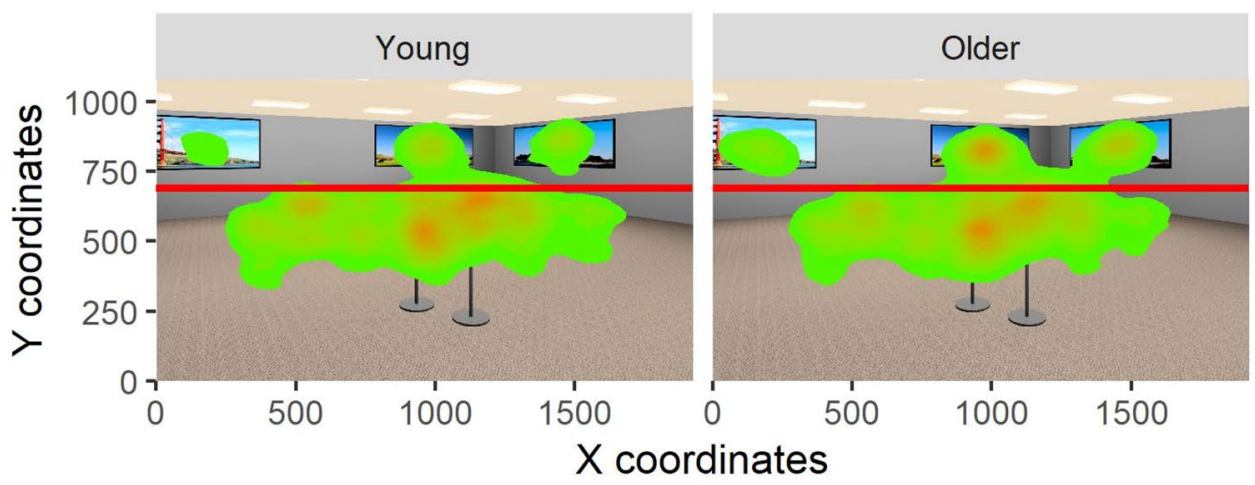

Dwell Time on the top IA across different Landmark Types at learning and test. We found strong positive correlations across all Landmark Types (No Landmarks: $\mathrm{R} 2=0.67, p<0.001$; Uninformative: $\mathrm{R} 2=0.88, p<0.001$; Informative: $\mathrm{R} 2=0.94$, $p<0.001)$. Those correlations suggest that participants are highly consistent in which stimulus features they gaze at during encoding and test.

\section{Discussion}

In the present study, we used eye-tracking to investigate age-related differences in visual encoding strategies employed for memorizing the locations of objects in a room. To do so, we explored if participants were able to 


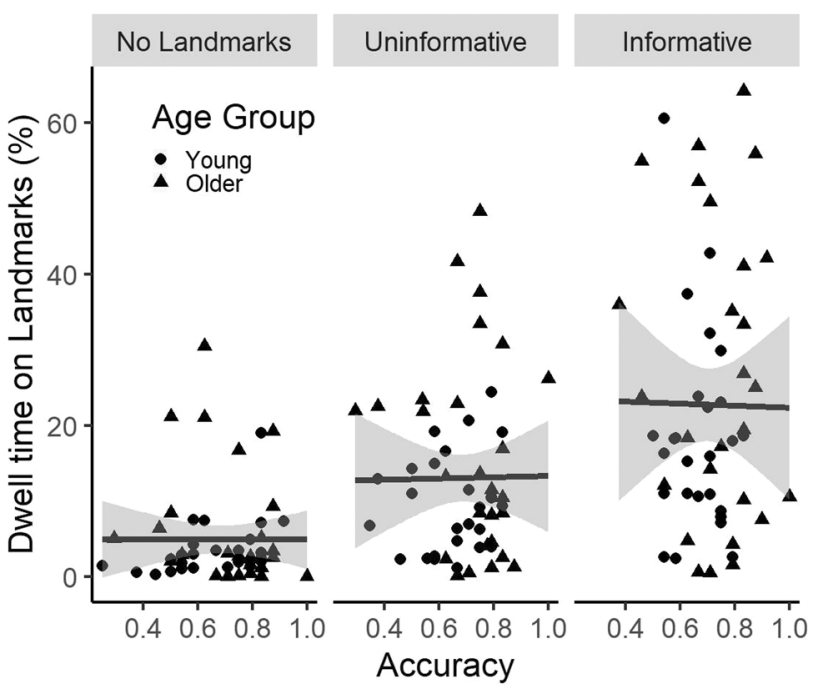

Fig. 6 Scatter Plot between Dwell Time on the top IA and Accuracy as a function of Landmark Type with regression line and CI (shaded area)

identify whether a spatial scene has changed following a perspective shift between encoding and test. The $30^{\circ}$ perspective shift was introduced to ensure that participants relied on spatial representations instead of solving the task by matching the visual image with a stored visual snapshot from encoding (Nardini et al., 2009). To investigate the effect of landmarks on encoding strategies, we also manipulated the availability and informative value of landmarks within the environment.

We found that overall, older adults took longer to respond. This increase in response times is consistent with findings that are widely reported in the cognitive ageing literature (Choice reaction time task: Woods et al., 2015; Memory: Hertzog et al., 2003; Language: Ratcliff et al., 2004a, b), and istypically attributed to decrements in speed of processing (Salthous, 1996; Salthouse \& Ferrer-Caja, 2003). We also found that the introduction of the perspective shift and the manipulation of object positions led to performance decrements in both age-groups. The availability and informativeness of the room-based landmarks did not affect task accuracy. Importantly, we found that when landmarks were presented, older participants spent more time than younger participants looking at the upper part of the display that contained the landmarks. This was particularly the case when the landmarks were informative.

Contrary to our expectations and previous place recognition research (Muffato et al., 2019; Hilton et al., 2020; Segen et al., 2020; Harley et al., 2007), there were no age-related differences in accuracy. However, it should be noted that we used an easier task than those used in previous studies, which could yield fewer problems for older adults. For example, the perspective shift we introduced was smaller than that of previous studies (Muffato et al., 2019; Montofinese et al., 2015; Segen et al., 2020). In addition, the scene at test could differ from the encoded only in terms of a change in the categorical relationship between objects. That is, in contrast to Segen et al. (2020), in the current study no changes in fine-grained spatial relationships between objects occurred. That the easier task may be responsible for the lack of agerelated deficits in task accuracy is in line with cognitive ageing research reporting greater age-related differences in performance with increasing task difficulty (Angel et al., 2016; Earles et al., 2004; Verhaeghen et al., 2006).

The lack of age-related performance accuracy differences in less demanding tasks can be explained by the compensation-related utilization of neural circuits hypothesis (ReuterLorenz \& Cappell, 2008). This hypothesis posits that under low task demands older adults can perform the tasks as well as young adults, supported by increased neural activations. However, when task demands increase, older adults' cognitive limits are reached resulting in performance declines that are typically accompanied by a reduction in activation in the relevant neural networks (Morcom \& Rugg, 2007; Angel et al., 2016). Thus, it is plausible that due to the relatively low task-demands in the current study, which are reflected in high performance across both age groups, older adults were able to carry out the task just as accurately as younger participants.

Consistent with our predictions, we found declines in accuracy in both age groups that were accompanied by an increase in response times when a perspective shift was introduced. This reduction in performance may have been driven by qualitative differences between trials that involved a perspective shift and those that did not. Specifically, without a perspective shift participant can refer to the representation of the learned scene from memory and use image matching to detect changes (Nardini et al., 2009). However, the introduction of the perspective shift required participants to engage in additional cognitive processing related to mental transformation in order to match the perspectives of the stored spatial configuration with the one presented at test (Hegarty \& Waller, 2004). However, it should be noted that the effect of the perspective shift was small, which is likely due to the relatively small perspective shift that we introduced.

Interestingly, there was a much more nuanced (if any) decline in accuracy or increase in response time in the Swap compared to the No Change condition when a perspective shift was introduced. To explain such findings, we turn to the response bias analysis which suggested that the introduction of the perspective shift increased the likelihood of participants responding that the object positions were "different". Thus, when a perspective shift was introduced in the Swap condition, this led to an increase in the number of correct responses albeit for the wrong reason. We believe that the 
increase of "different" responses after a perspective shift arises from the salient change in the visual input indicating that "something is different". However, if participants were solely responding to any change in the visual information between encoding and test, we expected them to perform below chance level in the No Change condition when a perspective shift was present. Yet, our participants were still able to perform well in this condition and their performance in the Swap condition with perspective shifts was not at the ceiling. This pattern of results demonstrates that participants were not solely relying on basic visual change detection but were instead using a spatial strategy to perform the task. Yet, they might have found it hard to inhibit the immediate response that the image is "not the same" when the perspective shift was introduced. The increase in performance in older adults with the introduction of the perspective shift in the Swap condition may thus be due to older adults experiencing even greater difficulty in inhibiting the response that the image is "not the same" when a perspective shift was present. Such difficulties are in line with age-related decline in executive functioning, in particular executive control (Braver \& West, 2008; Schretlen et al., 2000; Treitz et al., 2007).

Overall participants were more likely to make errors in the Swap condition than the No Change condition. To perform the task accurately participants in either condition had to bind an object's identity to its location (Postma et al., 2004; Waller, 2006). Previous research has shown that this is a cognitively demanding and error-prone process. For example, in place recognition studies participants were shown to be less accurate in detecting that a change has occurred when two objects swapped places compared to when a previously shown object was replaced by a new one (Hilton et al., 2020; Muffato et al., 2019). Similar results are reported in visuospatial working memory studies in which participants were asked to encode positions of abstract objects on a blank display. Participants were more likely to make swap errors, that is to place objects in the positions that were previously occupied by a different object (Pertzov et al., 2012, 2015).

Thus, the lower performance in the Swap condition can be explained by difficulties with binding objects to their locations, which prevents participants from accumulating information signalling that a change has occurred (Hilton et al., 2020; Muffato et al., 2019). Specifically, in the current task, the objects within the scene and their general configuration remained the same between learning and test. The only change introduced in the Swap condition is the position that each cluster occupied within that general configuration. Therefore, participants needed to remember the specific locations of each object cluster within that configuration to detect that a change has occurred.

In addition to comparing the behavioural performance of older and younger adults, another aim of this study was to use eye-tracking to investigate age-related differences in spatial encoding strategies and to study if such differences are driven by the information available within the environment. Firstly, we focused on general gaze parameters and found that older adults made more fixations that were shorter in duration as well as shorter saccades than young adults. While these results are consistent with those from a recent study using a similar place recognition task (Hilton et al., 2020), relating these general gaze measures to encoding strategies is difficult. We thus performed IA analysis which showed that gaze behaviour differed as a function of room type. As expected, we found that both age groups spent the lowest amount of time looking at the upper part of the stimuli in the No Landmarks condition in which there were no images on the walls of the room, followed by the Uninformative Landmarks condition, in which the images on the walls were all identical, and the Informative Landmarks condition in which each image was unique. These findings are consistent with results reported by Livingstone-Lee et al. (2011) who showed that participants quickly learned to adapt their gaze distribution in a virtual Morris water maze task based on the information that was available in the environment. Importantly, we found that compared to younger adults, older adults spent more time looking at landmarks in the Uninformative and Informative Landmarks conditions during encoding. A similar pattern was observed during the test phase in the Informative Landmarks condition.

A possible explanation for these age-related-differences in gaze behaviour is that older adults simply look around more due to a lack of a systematic encoding strategy. This can arise as a result of difficulties in selecting task-relevant information (Raptis et al., 2017). Given our results, however, it appears unlikely that older adults were randomly scanning the environment without a clear encoding strategy for several reasons: first, older adults solved the task as accurately as younger participants, which would not be possible without a clear encoding strategy. Second, we found that older adults' gaze behaviour changed as a function of the landmarks used. Specifically, older adults spent significantly more time looking at the upper part of the stimuli when landmarks were present and when these landmarks were informative, i.e. when they could be used to encode the spatial locations of the objects by relating objects to these room-based landmarks. Third, both younger and older adults adapted their gaze behaviour over the course of the experiment such that older adults spent less time fixating on uninformative landmarks across trials. Younger participants, on the other hand, spent less time fixating on informative landmarks across the trial. These changes in gaze behaviour over time are likely to reflect adaptations of encoding strategies with older adults learning to inhibit attending to uninformative information and younger participants focusing on 
encoding the relationship between objects even in the presence of informative landmarks.

Finally, gaze behaviour was highly consistent between learning and test, which suggests that participants, both young and older, attended to the same information during learning and test. It is possible that low-level properties of the stimuli (i.e. colour, intensity and orientation) contributed to such similarities in gaze behaviour through bottom-up control of attention (Itti, 2005), as similar visual information was presented at both learning and test. However, given that participants performed well on the task and made very few fixations at test, it is unlikely that the consistency between gaze behaviour at learning and test was solely driven by bottom-up processes. Instead, we suggest that participants relied on the information they encoded at learning to make decisions regarding whether or not the objects have moved at test. Together, these results suggest that gaze behaviour, in both younger and older adults, represents task and stimuli-dependent visual strategies that participants employed to solve the task.

Age-related differences in gaze behaviour may also be driven by older adults being distracted by salient, but taskirrelevant landmarks as a result of attention inhibition deficits (Hasher \& Zack, 1988; Healey et al., 2008, 2013). This account is partly supported by our findings as older adults spent more time than younger adults gazing at the uninformative landmarks. Notably, however, this did not affect their performance and can be explained by the relatively long encoding times that allowed participants to encode adequate task-relevant information even if they were briefly distracted.

An alternative explanation as to why older adults attended to uninformative landmarks (i.e. task-irrelevant information), is a more general age-related shift in the way they approach cognitive tasks. Zimmerman et al. (2011) suggested that older adults tend to implicitly encode all of the available information, regardless of its immediate utility. This is consistent with evidence showing that the inability to inhibit attention sometimes comes with benefits. Kim et al. (2007), for example, have shown that older adults display greater priming benefits when distractors on a previous task were used as primes in a problem-solving task. It is possible that the shift towards encoding irrelevant, as well as relevant information, stems from greater experience with real-world environments in which apparently task-irrelevant information often becomes relevant in the future (Kim et al., 2007; Zimmerman et al., 2011). For example, remembering extra landmarks in the environment could help to distinguish similar environments from each other. Such implicit shifts in encoding strategies may explain why older adults spent more time looking at extra information even if this information is not strictly necessary for solving the task at hand. However, such strategy shifts could lead to performance deficits in cognitively taxing situations, if older adults do not have enough resources to deal with the task at hand and if they are directing already limited resources to task-irrelevant information (Angel et al., 2016; Morcom et al., 2007; ReuterLorenz \& Cappell, 2008).

The idea that older adults have a greater preference than young adults towards encoding strategies that incorporate all available landmarks is consistent with results from research that employs diffusion modelling. Several studies document an age-related shift towards a more conservative response strategy whereby, compared to young adults, older adults prefer to accumulate more information before making decisions (Ratcliff et al., 2006, 2004a, b; Segen et al., 2020; Spaniol et al., 2006; Thapar et al., 2003). This explanation is also supported by our findings of longer response times in older adults which could be indicative of greater cautiousness.

Alternatively, the preference for attending to landmarks during encoding could be indicative of age-related differences in spatial encoding strategies. Specifically, older adults' may be more reliant on an encoding strategy in which they relate the positions of objects to landmarks, while younger participants focus on the local arrangement of objects and encode the spatial relationships between them. This interpretation is in line with our findings that older adults spent more time than younger adults looking at the landmarks during encoding, especially when these were informative. The differences in encoding strategies may represent an age-related shift towards the use of a categorical encoding strategy whereby participants bind an object to the nearest cue/landmark without the need to encode the exact metric relationship between the two. This shift may arise from difficulties in forming precise spatial representations. For example, previous visuospatial working memory research has shown that older adults were less precise in estimating previous locations of objects compared to younger adults, despite positioning the objects in the correct region of the stimuli (Nilakantan et al., 2018; Pertzov et al., 2015). Furthermore, in navigation, older adults show greater preference towards the use of beacon strategies (Wiener et al., 2013). Such strategies involve coarse categorical representations of locations in relation to environmental beacons or landmarks and may be preferred by older adults due to difficulties in formulating more precise representations.

Lastly, we did not find any relation between gaze behaviour and performance. This is not surprising as we found similar performance across different room types and across both age groups despite the presence of gaze differences. These results indicate that the current task can be solved equally well by focusing on objects and by relating the objects to landmarks (if they are available), with older adults showing a preference towards the latter. In addition, the lack of correlation between gaze and performance is consistent with our previous findings showing that the Swap condition 
could be solved either by looking around more or by having more focused gaze (Segen et al., 2020) outlining that coarse spatial representations can be formed using a wider range of encoding strategies and the available information.

To summarise, our results suggest that under specific conditions such as the presence of a relatively small perspective shift and the introduction of categorical changes within the scene, spatial memory is resistant to age-related changes as older adults perform the task as well as younger participants. Furthermore, we report an age-related shift in visual encoding strategy. Although we cannot completely rule out that these changes in gaze behaviour are driven by inhibitory control mechanisms, it seems highly plausible that older adults, who might be more distracted by the uninformative landmarks, employ an encoding strategy that relies on processing the categorical relationships between objects and room-based landmarks rather than forming fine-grain spatial representations.

Supplementary Information The online version contains supplementary material available at https://doi.org/10.1007/s00426-021-01495-5.

\section{Compliance with ethical standards}

Conflict of interest The authors declare that the research was conducted in the absence of any commercial or financial relationships that could be construed as a potential conflict of interest.

Ethical standards This study was carried out in accordance with the recommendations of the Research Ethics Code of Practice, Science, Technology and Health Research Ethics Panel at Bournemouth University with written informed consent from all subjects and has therefore been performed in accordance with the ethical standards laid down in the 1964 Declaration of Helsinki and its later amendments.

Open practices statement The datasets generated during and/or analysed during the current study are available in the Open Science Framework repository, https://osf.io/v4mwe/. This experiment was not pre-registered.

Open Access This article is licensed under a Creative Commons Attribution 4.0 International License, which permits use, sharing, adaptation, distribution and reproduction in any medium or format, as long as you give appropriate credit to the original author(s) and the source, provide a link to the Creative Commons licence, and indicate if changes were made. The images or other third party material in this article are included in the article's Creative Commons licence, unless indicated otherwise in a credit line to the material. If material is not included in the article's Creative Commons licence and your intended use is not permitted by statutory regulation or exceeds the permitted use, you will need to obtain permission directly from the copyright holder. To view a copy of this licence, visit http://creativecommons.org/licenses/by/4.0/.

\section{References}

Angel, L., Bastin, C., Genon, S., Salmon, E., Fay, S., Balteau, E., Maquet, P., Luxen, A., Insingrini, M., \& Collette, F. (2016).
Neural correlates of successful memory retrieval in aging: do executive functioning and task difficulty matter? Brain Research, 1631, 53-71. https://doi.org/10.1016/j.brainres.2015.10.009

Antonova, E., Parslow, D., Brammer, M., Dawson, G. R., Jackson, S. H. D., \& Morris, R. G. (2009). Age-related neural activity during allocentric spatial memory. Memory, 17(2), 125-143. https://doi. org/10.1080/09658210802077348

Baayen, R. H., Davidson, D. J., \& Bates, D. M. (2008). Mixed-effects modeling with crossed random effects for subjects and items. Journal of Memory and Language, 59(4), 390-412. https://doi. org/10.1016/j.jml.2007.12.005

Braver, T. S., \& West, R. (2008). Working memory, executive control, and aging. In F. I. M. Craik and T. A. Salthouse (Eds.), The handbook of aging and cognition (p. 311-372). Psychology Press

Earles, J. L., Kersten, A. W., Berlin Mas, B., \& Miccio, D. M. (2004). Aging and memory for self-performed tasks: effects of task difficulty and time pressure. The Journals of Gerontology Series B: Psychological Sciences and Social Sciences, 59(6), 285-293. https://doi.org/10.1093/geronb/59.6.P285

Hamburger, K., \& Röser, F. (2014). The role of landmark modality and familiarity in human wayfinding. Swiss Journal of Psychology. https://doi.org/10.1024/1421-0185/a000139

Hartley, T., Bird, C. M., Chan, D., Cipolotti, L., Husain, M., VargaKhadem, F., \& Burgess, N. (2007). The hippocampus is required for short-term topographical memory in humans. Hippocampus, 17(1), 34-48. https://doi.org/10.1002/hipo.20240

Hasher, L., \& Zacks, R. T. (1988). Working Memory, Comprehension, and Aging: A Review and a New View. Psychology of Learning and Motivation - Advances in Research and Theory, 22, 193-225. https://doi.org/10.1016/S0079-7421(08)60041-9

Healey, M. K., Campbell, K. L., \& Hasher, L. (2008). Cognitive aging and increased distractibility: Costs and potential benefits. Progress in Brain Research, 169, 353-363. https://doi.org/10.1016/S00796123(07)00022-2

Healey, M. K., Hasher, L., \& Campbell, K. L. (2013). The role of suppression in resolving interference: evidence for an age-related deficit. Psychology and Aging, 28(3), 721-728. https://doi.org/ 10.1037/a0033003

Hegarty, M., \& Waller, D. (2004). A dissociation between mental rotation and perspective-taking spatial abilities. Intelligence, 32(2), 175-191. https://doi.org/10.1016/j.intell.2003.12.001

Hertzog, C., Dixon, R. A., Hultsch, D. F., \& MacDonald, S. W. (2003). Latent change models of adult cognition: are changes in processing speed and working memory associated with changes in episodic memory? Psychology Aging, 18(4), 755. https://doi.org/10. 1037/0882-7974.18.4.755

Hilton, C., Muffato, V., Slattery, T. J., Miellet, S., \& Wiener, J. (2020). Differences in encoding strategy as a potential explanation for age-related decline in place recognition ability. Frontiers in Psychology, 11, 2182. https://doi.org/10.3389/fpsyg.2020.02182

Holmes, C. A., Newcombe, N. S., \& Shipley, T. F. (2018). Move to learn: Integrating spatial information from multiple viewpoints. Cognition, 178, 7-25. https://doi.org/10.1016/j.cognition.2018. 05.003

Inhoff, A. W., \& Radach, R. (1998). Definition and computation of oculomotor measures in the study of cognitive processes. In G. Underwood (Ed.), Eye guidance in reading and scene perception (pp. 29-53). Elsevier.

Kelly, D. J., Miellet, S., \& Caldara, R. (2010). Culture shapes eye movements for visually homogeneous objects. Frontiers in Psychology, 1, 1-7. https://doi.org/10.3389/fpsyg.2010.00006

Kim, S., Hasher, L., \& Zacks, R. T. (2007). Aging and a benefit of distractibility. Psychonomic Bulletin and Review, 14(2), 301-305. https://doi.org/10.3758/BF03194068

King, J. A., Burgess, N., Hartley, T., Vargha-Khadem, F., \& O'Keefe, J. (2002). Human hippocampus and viewpoint dependence in spatial 
memory. Hippocampus, 12(6), 811-820. https://doi.org/10.1002/ hipo. 10070

Livingstone-Lee, S. A., Murchison, S., Zeman, P. M., Gandhi, M., van Gerven, D., Stewart, L., Livingston, N., \& J. and Skelton, R. W. . (2011). Simple gaze analysis and special design of a virtual Morris water maze provides a new method for differentiating egocentric and allocentric navigational strategy choice. Behavioural brain research, 225(1), 117-125. https://doi.org/10.1016/j.bbr. 2011.07.005

Luis, C. A., Keegan, A. P., \& Mullan, M. (2009). Cross validation of the Montreal Cognitive Assessment in community dwelling older adults residing in the Southeastern US. International Journal of Geriatric Psychiatry, 24(2), 197-201. https://doi.org/10.1002/ gps.2101

Mathôt, S., Schreij, D., \& Theeuwes, J. (2012). OpenSesame: An open-source, graphical experiment builder for the social sciences. Behavior Research Methods, 44(2), 314-324. https://doi.org/10. 3758/s13428-011-0168-7

Meulenbroek, O., Petersson, K. M., Voermans, N., Weber, B., \& Fernández, G. (2004). Age differences in neural correlates of route encoding and route recognition. NeuroImage, 22(4), 1503-1514. https://doi.org/10.1016/j.neuroimage.2004.04.007

Moffat, S. D., Kennedy, K. M., Rodrigue, K. M., \& Raz, N. (2007). Extrahippocampal contributions to age differences in human spatial navigation. Cerebral Cortex, 17(6), 1274-1282. https://doi. org/10.1093/cercor/bhl036

Montefinese, M., Sulpizio, V., Galati, G., \& Committeri, G. (2015). Age-related effects on spatial memory across viewpoint changes relative to different reference frames. Psychological Research Psychologische Forschung, 79(4), 687-697. https://doi.org/10.1007/ s00426-014-0598-9

Morcom, A. M., Li, J., \& Rugg, M. D. (2007). Age effects on the neural correlates of episodic retrieval: increased cortical recruitment with matched performance. Cerebral Cortex, 17(11), 2491-2506. https://doi.org/10.1093/cercor/bhl155

Muffato, V., Hilton, C., Meneghetti, C., De Beni, R., \& Wiener, J. M. (2019). Evidence for age-related deficits in object-location binding during place recognition. Hippocampus, 29(10), 971-979. https://doi.org/10.1002/hipo.23099

Nardini, M., Thomas, R. L., Knowland, V. C., Braddick, O. J., \& Atkinson, J. (2009). A viewpoint-independent process for spatial reorientation. Cognition, 112(2), 241-248. https://doi.org/10.1016/j. cognition.2009.05.003

Nasreddine, Z. S., Phillips, N. A., Bédirian, V., Charbonneau, S., Whitehead, V., Collin, I., \& Chertkow, H. (2005). The Montreal Cognitive Assessment, MoCA: A brief screening tool for mild cognitive impairment. Journal of the American Geriatrics Society, 53(4), 695-699. https://doi.org/10.1111/j.1532-5415.2005. 53221.x

Nilakantan, A. S., Bridge, D. J., VanHaerents, S., \& Voss, J. L. (2018). Distinguishing the precision of spatial recollection from its success: Evidence from healthy aging and unilateral mesial temporal lobe resection. Neuropsychologia, 119, 101-106. https://doi.org/ 10.1016/j.neuropsychologia.2018.07.035

Nuthmann, A. (2017). Fixation durations in scene viewing: Modeling the effects of local image features, oculomotor parameters, and task. Psychonomic Bulletin and Review, 24(2), 370-392. https:// doi.org/10.3758/s13423-016-1124-4

Olk, B., \& Kingstone, A. (2015). Attention and ageing: Measuring effects of involuntary and voluntary orienting in isolation and in combination. British Journal of Psychology, 106(2), 235-252. https://doi.org/10.1111/bjop.12082

Pertzov, Y., Heider, M., Liang, Y., \& Husain, M. (2015). Effects of healthy ageing on precision and binding of object location in visual short-term memory. Psychology and Aging, 30(1), 26. https:// doi.org/10.1037/a0038396
Pertzov, Y., Miller, T. D., Gorgoraptis, N., Caine, D., Schott, J. M., Butler, C., \& Husain, M. (2013). Binding deficits in memory following medial temporal lobe damage in patients with voltage-gated potassium channel complex antibody-associated limbic encephalitis. Advance online publication. Brain: A Journal of Neurology, 136, 2474-2485. https://doi.org/https://doi.org/10.1093/ brain/awt129

Postma, A., Kessels, R. P. C., \& van Asselen, M. (2008). How the brain remembers and forgets where things are: The neurocognition of object-location memory. Neuroscience and Biobehavioral Reviews, 32(8), 1339-1345. https://doi.org/https://doi.org/10. 1016/j.neubiorev.2008.05.001

Raptis, G. E., Fidas, C. A., \& Avouris, N. M. (2017). On implicit elicitation of cognitive strategies using gaze transition entropies in pattern recognition tasks. In Proceedings of the 2017 CHI Conference Extended Abstracts on Human Factors in Computing Systems, 1993-2000. https://doi.org/https://doi.org/10.1145/3027063. 3053106

Ratcliff, R., Gomez, P., Thapar, A., \& McKoon, G. (2004a). A diffusion model analysis of the effects of aging in the lexical-decision task. Psychology and Aging, 19(2), 278-289. https://doi.org/10.1037/ 0882-7974.19.2.278

Ratcliff, R., McKoon, G., \& Gomez, P. (2004b). A diffusion model account of the lexical decision task. Psychological Review, 111(1), 159-182. https://doi.org/10.1037/0033-295X.111.1.159

Ratcliff, R., Thapar, A., \& McKoon, G. (2006). Aging, practice, and perceptual tasks: a diffusion model analysis. Psychology and Aging, 21(2), 353-371. https://doi.org/10.1037/0882-7974.21.2. 353

Reuter-Lorenz, P. A., \& Cappell, K. A. (2008). Neurocognitive aging and the compensation hypothesis. Current Directions in Psychological Science, 17(3), 177-182. https://doi.org/10.1111/j.14678721.2008.00570.x

Salthouse, T. A. (1996). The processing-speed theory of adult age differences in cognition. Psychological Review, 103(3), 403. https:// doi.org/10.1037/0033-295x.103.3.403

Salthouse, T. A., \& Ferrer-Caja, E. (2003). What needs to be explained to account for age-related effects on multiple cognitive variables? Psychology and Aging, 18(1), 91. https://doi.org/10.1037/08827974.18.1.91

Schretlen, D., Pearlson, G. D., Anthony, J. C., Aylward, E. H., Augustine, A. M., Davis, A., \& Barta, P. (2000). Elucidating the contributions of processing speed, executive ability, and frontal lobe volume to normal age-related differences in fluid intelligence. Journal of the International Neuropsychological Society, 6(1), 52-61. https://doi.org/10.1017/s1355617700611062

Segen, V., Avraamides, M. N., Slattery, T. J., \& Wiener, J. M. (2020). Age-related differences in visual encoding and response strategies contribute to spatial memory deficits. Memory and Cognition. https://doi.org/10.3758/s13421-020-01089-3

Spaniol, J., Madden, D. J., \& Voss, A. (2006). A diffusion model analysis of adult age differences in episodic and semantic long-term memory retrieval. Journal of Experimental Psychology: Learning Memory and Cognition, 32(1), 101-117. https://doi.org/10.1037/ 0278-7393.32.1.101

Thapar, A., Ratcliff, R., \& McKoon, G. (2003). A diffusion model analysis of the effects of aging on letter discrimination. Psychology and Aging, 18(3), 415-429. https://doi.org/10.1037/08827974.18.3.415

Treitz, F. H., Heyder, K., \& Daum, I. (2007). Differential course of executive control changes during normal aging. Aging, Neuropsychology, and Cognition, 14(4), 370-393. https://doi.org/10.1080/ 13825580600678442

Verhaeghen, P., Cerella, J., \& Basak, C. (2006). Aging, task complexity, and efficiency modes: the influence of working memory involvement on age differences in response times for verbal and 
visuospatial tasks. Aging, Neuropsychology, and Cognition, 13(2), 254-280. https://doi.org/10.1080/138255890969267

Waldron-Perrine, B., \& Axelrod, B. N. (2012). Determining an appropriate cutting score for indication of impairment on the Montreal Cognitive Assessment. International Journal of Geriatric Psychiatry, 27(11), 1189-1194. https://doi.org/10.1002/gps.3768

Waller, D. (2006). Egocentric and nonegocentric coding in memory for spatial layout: Evidence from scene recognition. Memory and Cognition, 34(3), 491-504. https://doi.org/10.3758/BF03193573

Waller, D. E., \& Nadel, L. E. (2013). Handbook of spatial cognition. American Psychological Association. https://doi.org/10.1037/ 13936-000

West, R. L. (1996). An application of prefrontal cortex function theory to cognitive aging. Psychological Bulletin, 120(2), 272. https:// doi.org/10.1037/0033-2909.120.2.272

Wiener, J. M., de Condappa, O., Harris, M. A., \& Wolbers, T. (2013). Maladaptive bias for extrahippocampal navigation strategies in aging humans. Journal of Neuroscience, 33(14), 6012-6017. https://doi.org/10.1523/JNEUROSCI.0717-12.2013

Wilcox, R. R., \& Keselman, H. J. (2003). Modem robust data analysis methods: Measures of central tendency. Psychological Methods, 8(3), 254-274. https://doi.org/10.1037/1082-989X.8.3.254

Woods, D. L., Wyma, J. M., Yund, E. W., Herron, T. J., \& Reed, B. (2015). Age-related slowing of response selection and production in a visual choice reaction time task. Frontiers in Human Neuroscience, 9, 193. https://doi.org/10.3389/fnhum.2015.00193 Zimmerman, S., Hasher, L., \& Goldstein, D. (2011). Cognitive ageing: a positive perspective. The paradoxical brain, 130-150

Publisher's Note Springer Nature remains neutral with regard to jurisdictional claims in published maps and institutional affiliations. 